\section{Variations des Précipitations atmosphériques d'après l'altitude dans le massif du Mont-Blanc}

Communication faite par M. P. Movgin, Inspecteur des Eaux et Forêts à Chambéry, au Congrès de Lyon de l'Association francaise pour l'avancement des Sciences, au cours de la séance du 7 août 1907 .

En 1902, le Service du Reboisemient de la Savoie a fait disposer sur le flanc S.-O. de la chaîne du Mont-Blanc, pour étuidier" l'importance des précipations à différentes altitudes, une série de rrécepteur's. Cés instruments, imaginés par M. Joseph Vallot et employés par lui dans son observatoire des Bosses, ont été légèrement modiflés. Alors qu'au MontBlanc, toutes les précipitations affectent la forme solide, la proportion de pluie augmente au fur et à mesure qu'on se rapproche du fond de la vallée. Chaque appareil est constitué par un cylindre de zinc de $0 \mathrm{~m}$. 20 de diamètre et de $2 \mathrm{~m}$. de hauteur. L'extrémité supérieure se termine par un trono de cône également en zinc, dont la petite base est un cer'cle de $0 \mathrm{~m}$. 08 de diamètre qui supporte un autr'e cylindre de $0 \mathrm{~m} .10$ de haut et de $0 \mathrm{~m}$. 08 de diamètre. La tranche libre du zinc est amincie et rendue presque coupante, de manière à diviser les flocon's de neige et à empêcher la formation de bour'relets neigeux.

L'extirémité inférieur'e du grand tube s'emboite sur un cône renversé dont le sommet, lerminé par une petite tubulure, s'arrête à $0 \mathrm{~m} .04$ du fond d'une cuvette. Cette cuvette, également en zinc, a la forme d'un tronc de cône, dont la petite base inférieure est un cercle de $0 \mathrm{~m}$. 20 de diametr'e et dont les flancs ont une inclinaison de 1/1. La grande base et dont les flancs ont une inclinaison de 11. La gr'ande base est constituée par un couvercle en zinc muni d'un rebord sur tout le pourtour et percé, en son milieu, d'une ouverture circulair'e de $0 \mathrm{~m}$. 22 de diamètre dont la périphérie est également garnie d'un rebord afin d'empêcher l'introduction, dans la cuvette, de toute eau qui ne proviendrait pas de l'appareil.

Tous ces récepteurs étant identiques, doivent donc se comporter de miême : chaque année, au momient du relevé des eaux rassembiées dans la cuvette, on prend, avec une feuille de papier, l'empreinte de l'ouverture. Au moyen d'un planimètre de précision, on détermine la surface réceptrice, et on tient compte des différences qui peuvent exister entre les anneaux pour le calcul de l'épaisseur de la lame d'eau annuelle.

Il importait d'éviter l'évaporation qui peut varier suivant l'altitude. A l'origtine, dans chaque cuvette, on a versé de l'essence de térébenthine jusqu'à l'extrémité de la tubulure qui termine le cône renver'sé. Cet hydrocarbur'e, peu soluble dans l'eau, ne bout qu'à $156^{\circ}$ sa densité est de 0,864 . Aussi devait-il, pensions-nous, exercert efficacement son action protectrice.

Mais, au cours d'expériences comparatives, faites avec divers récepteur's, à Chambéry, nous constatâmes, dans l'été 1904, que, dans un tube Vallot, il n'y avait plus d'essence. Pour déterminer lal quantité de ce liquide à verser' dans chaque instrument, de manière qu'il en existât toujour's audessus de l'eau, nous avons mis, dans une élprouvette en verre graduée, surmontée d'un entonnoir en zinc de section connue, assez d'essence pour former une lame de $0 \mathrm{~m}$. 035 d'épaisseur. Au bout d'un mois, les 73 pour 100 de l'essence s'étaient évaporés ; quinze jour's plus tard, vers le milieu de septembre, l'essence prit une couleur jaune sale en mêmie temps que l'eau recueillie, au-dessous, devenait blanchâtre. Puis brusiquement, toute trace d'essence sembla avoir disparu et un dépôt jaune, comme de la colophane gélatineuse, se précipita, au fond de l'éprouvette.

Il fallait chercher un autre isolant, nous nous sommes arrêtés à l'huile de vaseline, qui a les avantages d'être :

$1^{\circ}$ Insoluble dans l'eau ;

$2^{\circ}$ Plus légère que l'eaú ; $3^{\circ}$ Inaltérable, au moins pendant la période d'observation, qui ne s'étend pas au-delà d"une année ;

$4^{\circ}$ Incongelable aux températures ordinaires, ne prenant qu'aux environsi de $-25^{\circ}$ la consistance sirupeuse ;

$5^{\circ}$ Peu volatile. Ainsi, du 11 novembre au 31 juillet 1905 , il ne s'en est évapor'é que 56 centimètres cubes surv 120 placéls dans l'éprouvette, en verr'e, graduéé.

Commie les expériences ont porté sur la période comprise entre le $1^{\text {er }}$ octobre 1902 et le 3 octobre 1905 et que les relevés se sont faits, chaque année, au début d'octobre, on voit que les conditionsi ne sont pas demeurées identiques.

LES sections d'es récepteurs ne sont pas non plus demeurées les mêmes, car" le montage et le démontage des tubes, déforme toujour's, plus ou moins, les cylindres en zinc. Ces modifications, qui changent souvent en ovales les surfaces circulaires, peuvent avoir, pour conséquence, une facilité plus ou moins grande donnée à l'entrée des pluies et des neig'es. La correction que nous avons fait subir aux résultats trouvés, peut n'atténuer qu'une partie des résultats de la déformation. Tous les récepteurs ont été installés entrie les Houches ef le sommet de l'Aiguille du Goûter ; la distance horizontale, entre ces deux points quil ont entre eux une différence de niveau de $2.875 \mathrm{ml}$., n'atteint pas 5 kilomètres.

Le tableau suivant donne, avec les altitudes approchées des diverses stations, l'épaisseur' de la lame d'eau annuelle calculée en tenant compte de la surface réceptrice.

\begin{tabular}{|c|c|c|c|c|}
\hline \multirow[b]{2}{*}{ STATIONS } & \multirow[b]{2}{*}{ ALTITUDE } & \multicolumn{3}{|c|}{\begin{tabular}{cc} 
& \multicolumn{2}{c}{ HAUTEUR } \\
DE LA LAME D'EAU OB
\end{tabular}} \\
\hline & & $\begin{array}{c}\text { du } 1^{\text {er }} \text { octobre } \\
1902 \\
\text { au } 30 \text { septembre } \\
1903\end{array}$ & $\begin{array}{c}\text { du } 1 \text { et octobre } \\
1903 \\
\text { au } 24 \text { septembre } \\
1904\end{array}$ & $\begin{array}{c}\text { du } 25 \text { septembre } \\
1904 \\
\text { at } 3 \text { octobre } \\
1905\end{array}$ \\
\hline $\begin{array}{l}\text { Les Houches (ha- } \\
\text { meau de Pont). }\end{array}$ & 1,010 & $\begin{array}{c}\text { Milimètres } \\
481,9\end{array}$ & $\begin{array}{l}\text { Milimètres } \\
463,2\end{array}$ & $\begin{array}{l}\text { Milimètres } \\
8999,8\end{array}$ \\
\hline $\begin{array}{l}\text { Baraque inférieu- } \\
\text { re de la Greaz. }\end{array}$ & 1,540 & 492,5 & $(*)$ & $101^{\circ}, 8$ \\
\hline Barraque en bois & 2,100 & 319,4 & $(*)$ & 1308,8 \\
\hline $\begin{array}{c}\text { Plateau des Ro- } \\
\text { gnes............. }\end{array}$ & 2,550 & 1848,9 & 1755,7 & 2041,5 \\
\hline $\begin{array}{c}\text { Barraque de Pier- } \\
\text { re-Ronde....... }\end{array}$ & 2,850 & 448,1 & 531 & 782,4 \\
\hline $\begin{array}{c}\text { Laboratoire de } \\
\text { Tête-Rousse... }\end{array}$ & 3,185 & 368,2 & $3: 7,4$ & 492,9 \\
\hline
\end{tabular}

Ce tableau fait nettement ressortir un maximum, très accusé, au plateau des Rognes. La traduction graphique de ces résultats, absolument concordants, pendant 3 années, montre , d'un coup d'œil, que c'est aux environs de $2.500 \mathrm{~m}$. que les précipitationsi sont le plus abondantes ; un fait corr'obore cette conclusion : très souvent, dans nos ascensions et pendant nos séjours à Tête-Rousse, nous avons vu émerger la Crête des Rognes au-dessus des nuages, par suite, se trouver au-dessus de la zone des précipitations. Rien d'étonnant que le plateau des Rognes qui, dans ces cas, est toujour's caiché par les brouillar'ds, reçoive une lame d'eau bien supérieur'e à celle qui tombe sur le plateau de Pierre-Ronde. Il ne faut pasi alcicepter, non plus, les chiffres donnés dans le tableau, en valeur absolue. S'ils sont comparables, entre eux, dans la même année, il semble bien qu'il ne faille pas les mettre en parallèle avec les r'enseignements fournis part le pluviomètre. En ruisselant le long des parois du tube Vallow, les gouttes d'eau; au moment chaud de la saison chaude, mouillent une superficie considérable de métal rugueux, s'évaporent, et il existe, de ce chef, une déper'dition sérieuse. Ainsi, par des expériences compar'atives, le tube Vallot n'aurait donné,pendaint un an (1 ${ }^{\text {er }}$ octobre $1902-30$ septembre 1903) que 55,6 pour 100 de la lame d'eaiu recueillie

(*) Appareils détruits par des chutes de pierres. 
au pluviomètre ; d'autres expériences sur les mensurations de neige, pour l'hiver 1902-190s, ont donné 68,9 p.our 100 comme rendement du même tube, toujours par rapport au piuviomètre.

Il restie à étudier la nature des précipitations qui affectent tantôt la forme solide et tantiot la forme liquide. Il est clair, qu'au fur' et à mesure qu'on s'élèvera, les neiges seront plus fréquentes et plus abondantes, mais il est bien difficile d'avoir des chiffres exacts dans des régions où l'on ne séjourne gueir'e et où piersonne ne se trouve à point pour mesur'er l'épaisseur et la densité des couches neigeuses.

Même en partant de la lame d'eau recueillie dans la vallée, on ne saurait apprécier la proportion de neige qui tombe aux diver's étages de la montagne. Si l'on se base sur la tempér'atur'e qui décroît de $1^{\circ}$ par $170 \mathrm{~m}$. ou $180 \mathrm{~m}$. d'altitude, on pourrait, connaissant le degré thermique de Chamonix, évaluer la tempérlature au même moment aux diverses stations. Mais cette détermination n'aurait rien de précis, en raison même des couranls aériens, très variables, qui peuvent régner du haut en bas du versant, surtout lor'squ'on se trouve, comme c'est ici le cas, au point de confluence de 2 vallées : celle de Chamonix et celle de Montjoie.

En supposiant même qu'on puisse ainsi calculer exactement la température en un point et à un moment donnés, devra-t-on conclure à une chute de neige si le thermomètre mar'que $0^{\circ}$ en cette station et s'il pleut en bas ? Ce serait une grave erreur. D'après les observations niviométriques faites par" le Service de Reboisement, depuis décembre 1899, on voit qu'il peut neiger par' des températures bien supémieures à $0^{\circ}$. Ainsi, à Chambéry, il a neigé à une température de $+7^{\circ}$; à Bessans, à Sallanches, par +10 ; dans les postes militaires de la Redoute Ruinée et du Col de Sollières, on a observé đe la neig€ dans l'hiver 1904-1905, par des températures de $+12^{\circ}$ et $+13^{\circ}$.

En 7 hiver's, sur 31 sitations d'observations, on n'a noté qu'une seule de ces stations où il a neigé un hiver, sans que le thermomètre ait dépassé $0^{\circ}$ (Les Chapieux, 1899-1900).

Inversemient, il peut tomber de la pluie au-dessous de $0^{\circ}$. G'est ainsi, qu'à Tête-Rousse, il a plu le 3 octobre 1901 par - $1^{\circ}$, le 28 juillet 1903 , par $-0^{\circ} 5$, le 2 octobre 1903, par $-1^{\circ}, 5$; on voit que, dans ces conditions, on ne peut tirer des conclusions sur la nature des précipitations d'après la seule connaissance du degré thermique.

Aux Houches, l'une de ces stations, pendant les 3 hivers considérési, les mensurations de neige ont donné les résultatis suivants :

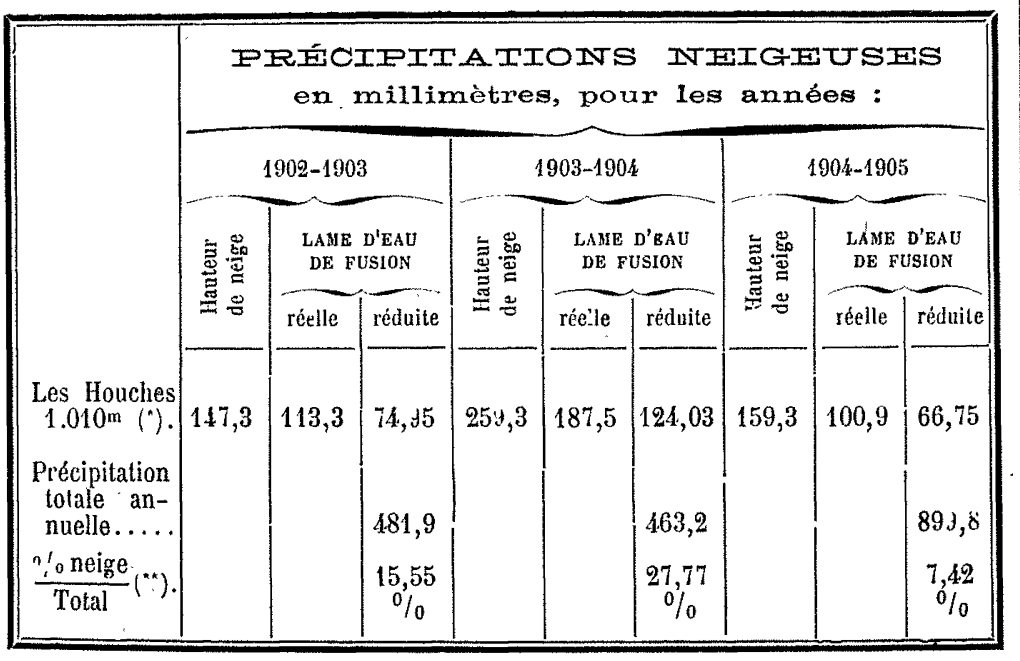

Afin de pouvoir comparer les données obtenues à l'aide de la planche niviométrique avec celles fournies par le tube Vallot des Houches, nous res avons réduites en les inultipliant par 0,6615. Ce facteur de conver'sion est le résultat

(*) Le coefficient de réduction est de 66.15 pour 100 .

(*) Soit, en moyenne, 14.4 pour 100 . d'expériences poursuivies, à Chambéry, pendant les hivers 1901-1902 et 1902-1903 avec ces deux instruments. On tiendra ainsi compte, dans une certaine mesure, des pertes provenant de l'emploi du tube Vallot, et si les proportions entre la quantité réduite d'eau de fusion de la neige tombée et la précipitation totale annuelle entre le $1^{\text {er }}$ novembre 1903 et le 3 novembr'e 1905, ne sont pas mathématiquement exactes, elles se rapprochent cependant de la vérité.

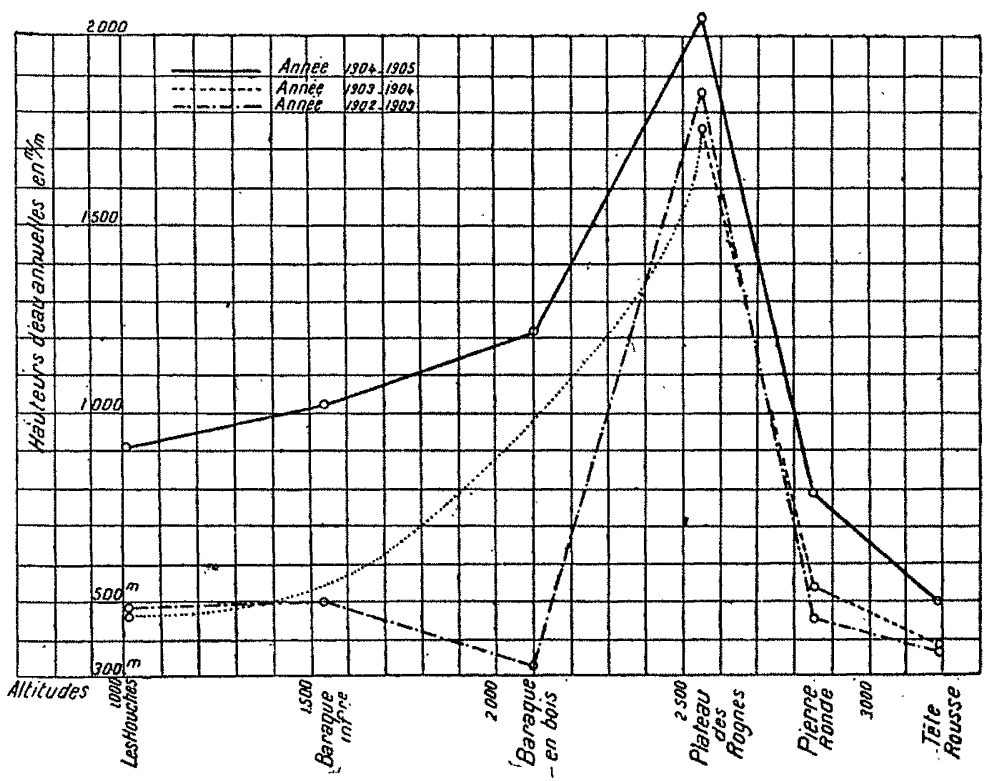

Variations de Ia lame d'eau annuelle, d'après l'altitude, dans le massif du Mont-Blanc (1er octobre 1902, 3 octobre 1905).

A Tête-Rousse (3.180 m.), le service fornestier a exécuté, depuis 1901, des observations météorologiques, malheureusement, il n'a exécuté, au début, aucune mensuration pluviométrique.

En radison de l'altitude considérable de la station, les précipitations y affectent normalement la forme solide $6 t$ les relevés se font sur 4 tables et sur une série de 35 piquets placés sur le glacier.

Ce n'est guère qu'à partir du début de juillet, jusqu'aux pr'emier's jour's d'octobre, qu'il est possible de séjourner dans ces hautes régions.

Durant l'été 1903 , on a noté :

$\left.\begin{array}{crr}6 & \text { jours de pluie en juillet...... } \\ 8 & - & \text { en aout....... } \\ 4 & - & \text { en septembre. } \\ 2 & \text { en octobre sur } \\ & \text { les treize premiers jours..... }\end{array}\right\} \begin{aligned} & \text { Soit au total, } \\ & 28 \text { jours de } \\ & \text { pluie. }\end{aligned}$

La durée de ces pluies a été généralement très courte, surtout à la fin d'août et en septembre.

Ce n'est qu'en 1905 qu'on a commencé à mesurer les quanlitiés de pluie tombées à T'ête-Rousse ; on a trouvé $45 \mathrm{~mm}$. pour les mois de juillet, aout et septembre ; si l'on fait subir la réduction de 66,15 p. 100 , on arrive au chiffre de $29 \mathrm{~mm}$. 77 et, par suite, le rapport de la pluie tombée à la précipitation totale, pour 1905, sera de :

$$
\frac{29.77}{492.9}=6,03 \text { pour } 100
$$

On ne peut, évidemment, tabler sur une observation unique qui ai, cependant, l'avantage de donner' une idée de la faible imiportance des pluies à l'altitude de $3.185 \mathrm{~m}$.

Ainsi qu'on vient de le voir, les observations sur les divers éléments météorologiques, sur le flanc sud-Ouest du miassif du Mont-Blanc, ne sont pas encore très avancées.

De la constance remarquable du maximum de précipitations à $2.550 \mathrm{~m}$., on peut, avec vraisemblance, conclure à 
l'existence de ce maximum ; les proportions de pluie ou de neige auront besoin d'être plus suivies ; l'étude des variations thermiques, aux différents étages de la montagne, sera entreprise, pendant la belle saison, cette année même.

Il ne serait pas moins intéressant de rechercher, au moyen de baromètres enregistreur's, jusqu'a quel niveau se font sentir' les mouvements tourbillonnaires de l'atmosphère, si violents dans les régions supérieures et qui sont déjà atténués à la hauteur de Tête-Rousse, mais nous n'avons pas les instruments nécessair'es, non plus que les hvgromètres enr'egistreurs.

Il appartiendra à l'Administration des Eaux et Forêts, qui a déjà tant fait, comme vous venє\% de le voir, pour favoriser los observations en haute montagne, de dire si elle veut étendre encore, ce qui est tr'ès souhailable, le champ de ses investigations et entreprendre ces nouvelles études.

\section{Le Reboisement en Savoie}

Communication faitc par M. Mougrn, Inspecteur des Eaux et Forèts, au Congrès de Lyon de l'Associalion francaise pour l'avancement des Sciences, au cours de la séance du 3 août 1906.

On ne discule plus l'opportunité du reboisement en mon. tagne. Lo retour périodicue des inondations de la Loire et surtoub du. Rhône et de ses affluents ont misi celte question à l'ordrte du jour". Depuis longtemps, on a signalé la dégr'alation progr"essive des Alpes, les ravages de leurs torrents et la répercussion fàcheuse de la dénudation des pentes sur le l"égime des eaux el la slabilité du lit des rivières. Administrateur's, ingénieurs el lorestiers étudièrent les causes des débordements, des érosions des versants, de l'enlisement des villages et des cultures.

Ein 1841, Surell, ingénieur des Ponts et Chaussées du département des Hautes-Alpes, dans un livre qui fait autorité, a démontrél les quatre propositions suivantes :

$1^{\circ}$ La présence d'une forèt sur un sol empèche la formation des torrents'

$2^{\circ}$ Le débcisement d'une forèt livre le sol en proie aux torrents ;

$3^{\circ}$ Le dévelcppement des for'êts provoque l'extinction des torrents ;

$4^{\circ}$ La chute des forêts redouble la violence des torrents, peut mème les faire renaître.

Dans son "Eitude sur les travaux de reboisement et de gazonnement des monlagnes ", parue en 1878, un forestier, Demontzey, en exposant les móvens pratiques de rétablir la forêt, là où elle avait disparu, écrivait :

"Il est indispensable de bien préciser le but qu'on se propose en entreprenant une semblable opération. Ce but est la création d'une végétation ligneuse qui répond aux conditions suivantes :

'" $1^{\circ}$ Posséder des racines assez puissantes, pour enserr'er le sol dans leurs innombrables réseaux, le rendre au besoin plus perméable et le priotégter contre l'entraînement.

" $2^{\circ}$ Présenler" un couvert assez complet pour abriter la surface contre les influences météorologiques;

" $3^{\circ}$ Fourmir" un humus de plus en plus abondant, appelé, d'une part, à fertiliser le sol et à augmenter la puis'sance de la végétation et, d'autre part, ì favoriser le ralentissement et la régularisation du débit des eaux pluviales ou des neiges fondant à sa surface ;

" $4^{\circ}$ Maintenir sans interruptions momentanées et perpétuellement, ces salutair'es effets et les développer avec l'aide du temps.

"La futaie, seule, est capable de satisfaire, sans restrictions, à l'ensemble de ces conditions ; c'est donc sa création et son développement qui doivent ètre le but final du reboisement $n$.
Depuis l'époque où lurent écrites ces lignes, on arriva a démontrer, par des séries d'observations, que le couvert des résineux arrêtait environ la moitié de la lame d'eau tombée ; que l'humus absorbait jusqu'à deux fois et demie son poidis des eaux atmosphérriques qui lui parrvenaient et qu'enfin les racines faisaient baisser la nappe phréatique plus bas sous bois que hor's bois, assainissant, asséchant ainsi la conche supérieur'e du sol. Pair respiration, les arbres renvoient dans l'atmosphèr' $€$. une partie de cette humidité, d'où un climat plus égal, des pluies plus fréquentes.

Enfin la présence de rideaux boisés brise la violence des vents qui dessèchent les vallées comme la Maurienne ou qui descendent en rafales puissantes des cols élevés des Alpes, du P'elit-Saint-Bernard, par exemple.

Mais alor's qu'on avail attaqué énergiquement le phénomène torrentiel dans les Hautes et les Basses-Alpes, dans l'Isère, dans la Dr'òme, il semblait qu'on eût oublié les départements de la Savoie. Pourtant, immédiatement avant et après l'annexion de 1860, des inondations, des laves (on appelle amsi des coulées boueuses produites par. les torrents), avaient causé dans la région de terribles dommiages el avaient eu leur r'éplercussion sur l'Isère et sur le Rhôn* lui-même.

Le $1^{\text {er }}$ novembre 1859 , c'est l'Isère, l'Arc, l'Arly qui débordent, rompent leurs digutes et détreminent la submersion du Grésivaudan et d'une partie de la ville de Grenoble.

En 1866, le 25 septembre, c'est le tor'rent de l'Envers en Maurienne qui barre l'Ar'c el amène une débâcle formidable. "LArc, dit dans son rapport, le Ministre des Travaux Publics, a éprouvé une crue telle qu'on en a jamais signalé, même aux époques les plus reculées. Fn quelques heures, la vallée étail dévastée, la route impériale du Mont-Cenis clétruite sur plus de six kilomètres de longueur, etc..." ".

Un peu plus tard, le 20 juillet 1871 , c'est le ruisseau de Saint-Julien qui coupe encore la route nalionale de Paris à Turin et interrompt, pendant vingt-neuf jours, tout trafic sur la voie ferrée du Rhòne au Mont-Cenis. Une nouvelle lave du même torr'enti ar"ête également la circulation pendant dix-neuf jours : du 24 juillet au 12 août 1872 .

En 1875, le 18 janvier, la ville de Chambéry est inondée par lGs eaux du torrent de Leysse.

Comme si ce n'était pas assez, des torrents nouveaux naissent, se développent. C'est la Grollaz, inoffensif ruisseau, qui vient recouvrir de matériaux la voie ferréie près de Saint-Michel-de-Maurienne ; c'est, en Tarentaise, le Sécheron qui, dès 1868, menace d'engloutir un des hameaux de la commune de Le Bois ; ciest le Nant-Trouble, à Ugines, de 1877 à 1882, qui char"rie dans la plaine des Corroies plus de 1 million de mètres cubes des terres arrachées à la montagne du Siapey et étale ses déjections jusque sur la chaussée de la route départementale d'Ugines à Seyssel, qui cependant était établie en remblai.

La lèpr"e torrentielle allait augmentant tous les jours, car la Savoie, jadis couverte de vastes forềts, se déboisait chaque jour davantage.

Dans le cours du XIX ${ }^{e}$ siècle, la Tarentaise voit disparâ̂tire 11,5 p. 100 de ses massifs communaux et la Maurienne 38 p. 100. Au pied du Mont-Cenis, le seul canton de Lanslebourg a perdu 41 p. 100 de sa surface forestière !

Il était dono excessivement urgent de réagir. En 1880, le service forestier commence la correction du torrent de la Grollaz, en Maurienne. Mais, devant l'opposition que rencontra le reboisement, il doit se borner aux travaux d'art, barrlages et drains destinés à consolider temporairement le sol.

Six ans plus tard, l'Etat acquiert le glissement de Sécheron, l€ draîne et exécute ses premières plantations. Ce n'est qu'en 1894, que l'Administration des Eaux et Forêts acquiert 1.294 hectar'es dans les vallées de l'Isère et de. l'Arly et 2.518 hectar'es dans celle de l'Arc, en amont de Saint-Jeande-Maurienne. Malheureusement, ces surfaces fort insuff- 